\title{
Review of: "Asymmetric biomimetic transamination of $\alpha$-keto amides to peptides"
}

\author{
wanbin zhang ${ }^{1}$ \\ 1 Shanghai Jiaotong University
}

Potential competing interests: The author(s) declared that no potential competing interests exist.

Peptides are biologically active compounds which are related to various cell functions in organisms. The development of efficient and convenient synthesis methods has attracted chemists' interest. This work reported an asymmetric transamination of $\alpha$-keto amides catalyzed by a bioinspired $\mathrm{N}$-quaternized chiral pyridoxamine, contributing an interesting alternative method for the synthesis of peptides. The pyridoxamine catalyst is the key for the transformation, which dominates the absolute configurations of the peptide products. A pair of diastereomers both can be obtained with high enantiopurity from a same chiral $\alpha$-keto amide by switching the configuration of the pyridoxamine catalyst, making the reaction is especially attractive for the synthesis of peptides made of unnatural amino acids. 